\title{
Insulin-induced Lipohypertrophy in Patients with Type 1 Diabetes Mellitus Treated with an Insulin Pump
}

\author{
Damian Ucieklak $\mathbb{D}^{1,2}$ Sandra Mrozinska ${ }^{10},{ }^{1,2}$ Aleksandra Wojnarska ${ }^{1,2,3}$ \\ Maciej T. Malecki $\left(\mathbb{D},{ }^{1,2}\right.$ Tomasz Klupa $\mathbb{D}^{1,2}$ and Bartłomiej Matejko ${ }^{10}{ }^{1,2}$ \\ ${ }^{1}$ Department of Metabolic Diseases, Jagiellonian University Medical College, Krakow, Poland \\ ${ }^{2}$ University Hospital, Krakow, Poland \\ ${ }^{3}$ Department of Radiology, Jagiellonian University Medical College, Krakow, Poland \\ Correspondence should be addressed to Bartłomiej Matejko; b.matejko@uj.edu.pl
}

Received 1 October 2021; Revised 23 December 2021; Accepted 27 December 2021; Published 24 January 2022

Academic Editor: Dario Pitocco

Copyright (c) 2022 Damian Ucieklak et al. This is an open access article distributed under the Creative Commons Attribution License, which permits unrestricted use, distribution, and reproduction in any medium, provided the original work is properly cited.

\begin{abstract}
Background. Lipohypertrophy (LH) of subcutaneous tissue is an insulin-induced complication occurring in patients with diabetes. We aimed to define the prevalence of LH and identify its risk factors in type 1 diabetes (T1DM) patients treated with continuous subcutaneous insulin infusion (CSII). Materials and Methods. The study included 79 consecutive CSII-treated T1DM patients. The diagnose of LH was based on ultrasonography (US) as a reference method, physical examination was also performed. Clinical characteristics were available from the medical records. Results. The median age of patients was 28 years (interquartile range [IQR], 24-30.5) with a body mass index (BMI) of $24.5 \pm 3.5 \mathrm{~kg} / \mathrm{m}^{2}, \mathrm{HbA}_{1 \mathrm{c}} 7.1 \%$ (IQR, 6.7-8.1), T1DM duration 15 (9-20) years, and CSII use duration of 8 year (IQR, 5-11). LH was detected by US in 75 (94.9\%) patients. This value was much higher than this obtained by visual assessment $(n=39,49.4 \%)$ or palpation $(n=59,74.7 \%)$. In univariate analyses, the following risk factors for occurrence of 5 and more LH lesions were identified: the ratio of insulin dose to body mass exceeding $0.7 \mathrm{IU} / \mathrm{kg}$ (OR, 3.69; 95\% CI, $1.43-10.01)$ and the total daily insulin dose (OR, 1.05; 95\% CI, 1.02-1.09). A higher dose of insulin per kg remained a significant risk factor of LH amount in multivariate analysis. Conclusion. This selected T1DM cohort treated with CSII had a very high prevalence of LH. US assessment should be considered as a reference method for LH screening in T1DM patients. The identified risk factors for the number of $\mathrm{LH}$ lesions were related to insulin dosing.
\end{abstract}

\section{Introduction}

Lipohypertrophy (LH) is a common complication of insulin therapy in subcutaneous tissue observed in patients with diabetes [1]. It manifests as soft benign nodules on the skin surface [1]. LH occurrence is associated with the lipogenic action of insulin at the site of injection and repeated trauma related to performing insulin injections at the same site [2]. Several risk factors of LH such as a needle reuse, a lack of rotation of injection sites, a small size of rotation area, a high daily insulin dose, a long duration of insulin treatment, a high body mass index (BMI), poor glycemic control, and low level of patient education [3-6] were reported from clinically diverse diabetic cohorts. The risk of LH in patients treated with multiple daily insulin injections was reported to be lower when using insulin analogs than human insulin [2]. The insulin absorption from affected areas is impaired and can be probably attributed to the local inflammatory reaction, increased local fibrosis, and decreased vascularity [7-9].

Of note, $\mathrm{LH}$ has been linked to poor glycemic control as well as a higher frequency of unexplained episodes of hypoglycemia as well as an increased variability in glucose levels and ketoacidosis [10-13]. What is more, injecting insulin into LH lesions can lead to an inappropriate increase in insulin dosing and-consequently higher costs of insulin 
therapy $[14,15]$. Omitting during injections places affected by $\mathrm{LH}$ seems to decrease total insulin dose and improve glycemic control [16-18].

Data on the prevalence of $\mathrm{LH}$ varies significantly, depending on the characteristics of the study group, method of insulin therapy, and methodology used for LH diagnosis $[14,19-21]$. Ultrasonography (US) was recognized to be a useful diagnostic method for the assessment of $\mathrm{LH}$ $[7,20,22]$. The typical ultrasound echo representation of $\mathrm{LH}$ is an increased echogenicity spot in diffuse areas of the subcutaneous tissue where insulin is injected. However, LH can sometimes manifest as clearly defined nodules with circumscribed margins [20]. Hyperechogenic areas have a prevailing fibrotic component while hypoechogenic ones have edema or fluid components without fibrotic segments [7]. Another important feature of LH nodules that helps differentiate LH lesions from other benign superficial masses like lipomas, hematomas, or fluid-filled cysts [22] is the lack of a capsule or vascularity. In this study, we aimed to define the prevalence of LH in T1DM patients treated with continuous subcutaneous insulin infusion (CSII) on basis of US as a reference method. A second goal was to identify risk factors.

\section{Materials and Methods}

We evaluated 79 consecutive T1DM patients treated with an insulin pump from the outpatients of Department of Metabolic Disease, University Hospital in Krakow (tertiary diabetes reference center). The following inclusion criteria must have been fulfilled: (1) diagnosis of T1DM according to WHO criteria [23]; (2) age at the examination 18-50 years; (3) insulin pump use for at least 6 months; and (4) having given written informed consent. Exclusion criteria were as follows: (1) pregnancy, (2) $\mathrm{BMI}>32 \mathrm{~kg} / \mathrm{m}^{2}$; (3) atopic dermatitis; (4) subcutaneous administration of medicines other than insulin in the last 6 months; and (5) presence of advanced diabetes complications such as end-stage renal disease or loss of vision.

Clinical characteristics of the patients were obtained from their medical records. The data from the insulin pump reports and glucometers for the last 14 days before the examination was collected. Patients were asked to complete a questionnaire about the frequency of changing the infusion sets.

All patients were examined for the presence of LH by the physical examination through the visual assessment and palpation and with US (Acusone, Siemens) scanning. US examination of all patients was performed using one of the two sonographers participating in this study. On US, LH was diagnosed based on previously described criteria [22]. These involved lesions characterized by: (a) localization in the subcutaneous tissue between the epidermis and the muscle layer in the area of the insulin injections; (b) clear circumscription either by hyperechoic foci with defined borders or a nodular shape with a hypoechoic halo; (c) heterogeneous echotexture versus surrounding tissue; (d) distortion of surrounding connective tissue; and (e) absence of a capsule or vascularity.
The number and localization of LH lesions that were found in each examination and the largest dimension of the biggest LH lesion on US were recorded. Considering the limitations of LH severity assessment based only on the number of lesions, we also proposed a new LH severity scale considering both the amount and size of LH lesions. The details of scoring were as follows:

Number of LH lesion: none $=0$ points; 1 lesion $=1$ point; 2-3 lesions $=2$ points; $4-7$ lesions $=3$ points; and $\geq 8$ lesions $=4$ points.

Size: no $\mathrm{LH}$ lesion $=0$ points; the maximal diameter of the biggest $\mathrm{LH}$ lesion $\leq 1 \mathrm{~cm}=1$ point; $>1$ and $\leq 2 \mathrm{~cm}=2$ points; $>2$ and $\leq 3 \mathrm{~cm}=3$ points; and $>3 \mathrm{~cm}=4$ points.

The sum of the number and size points together categorized LH into four stages of advancement: no $\mathrm{LH}=0$ points; mild $\mathrm{LH}=1-2$ points; moderate $\mathrm{LH}=3-5$ points; advanced $\mathrm{LH}=6-8$ points.

Visual inspection and palpation of insertion sites were performed in patients in both a standing and supine position. In the visual assessment, lesions that protruded above the skin surface were rated as LH. Other skin lesions like spot marks (i.e., slight redness, scars) in areas of the former cannula implantation in the level of skin surface were not counted as LH. The definition of LH in palpatory examination was based on earlier publications [22]. Briefly, LH was defined as discrete palpable dermal nodules or swellings of variable consistency. These were either thickened, had a 'rubbery' texture, or felt firm and were located within the subcutaneous tissue at insulin injection sites. The term "subclinical LH" was reserved for LH lesions detected on US which were not detected by visual or palpation method [22]. All participants provided written informed consent in accordance with the Declaration of Helsinki.

\section{Statistical Analysis}

Continuous variables with normal distribution were expressed as the mean \pm standard deviation (SD). Continuous variables not normally distributed were presented as median and interquartile range (IQR). The Shapiro-Wilk test was performed to assess whether a variable was normally distributed. The differences between two groups were assessed using the Student's or the Welch's $t$-test for normally distributed continuous variables; otherwise the Mann-Whitney $U$ test was conducted. The Chi-square or Fisher's exact test was used to determine whether there is a significant association between two categorical variables. Three groups were compared using the Kruskal-Wallis test as non-parametric alternative to the one-way ANOVA test. The Pairwise Wilcoxon Rank Sum Test with Bonferoni correction was performed to calculate pairwise comparisons between group levels with corrections for multiple testing.

Uni- and multivariate logistic regression models were used to identify the risk factors of the number and the severity of LH lesions based on the proposed scale. In univariate models, we used the following variables: age, gender, BMI, T1DM duration, time on CSII, changing infusion sites regularly (Yes/No), changing infusion set every three days or more frequently (Yes/No), length of cannula 
above or equal to $9 \mathrm{~mm}, \mathrm{HbA}_{\mathrm{lc}}$ level, daily dose of insulin, and daily insulin dose above $0.7 \mathrm{IU} / \mathrm{kg}$. A daily insulin dose above $0.7 \mathrm{IU} / \mathrm{kg}$ was chosen because this value constituted a mean insulin requirement in a large group of adult T1DM patients treated with CSII [24]. Variables with $p$-values less than 0.10 in the univariate model were used in multivariate analysis.

The Cohen statistic was used to assess the inter-observer agreement between LH lesions detected on US and physical examination. The McNemar test was used to check if two methods are equal in LH detection. We also assessed sensitivity and specificity of physical examination against US as a gold standard approach. The level of significance for the two-sided tests was set to below 0.05 . Statistical analyses were performed using Statistica 12.5 software (StatSoft Inc., Tulsa, Oklahoma, United States) and R ver. 3.6.3 [25].

\section{Results}

4.1. Patient Characteristics. We included 79 consecutive T1DM patients [39, (49.4\%) women]. Detailed clinical characteristics for the entire group as well as for subgroups with in-target of $\mathrm{HbA}_{1 \mathrm{c}}<7 \%$ and above-target $\mathrm{HbA}_{1 \mathrm{c}}$ levels are shown in Table 1 . This includes a median age of 28 (IQR 24-30.5) years, a $\mathrm{HbA}_{1 \mathrm{c}}$ level of 54 (IQR 51-65) $\mathrm{mmol} / \mathrm{mol}$, $7.1 \%$ (IQR 6.7-8.1\%), BMI $24.5( \pm 3.5) \mathrm{kg} / \mathrm{m}^{2}$, T1DM duration 15 years (IQR 9-20), and duration of insulin pump use of 8 years (IQR 5-11). Only six patients presented chronic diabetic complications (three cases of retinopathy, three of polyneuropathy, and one of diabetic kidney disease). At this sample size, the proportion of patients with $\geq 5 \mathrm{LH}$ lesions on US was not statistically different between the two T1DM subgroups defined on the basis of glycemic control. However, this percentage was numerically higher in patients with the HbA1c level above the glycemic target (58.3 vs. $48.3 \%$ ).

Additionally, we also collected data referring to the type of insulin, insertion cannula, pump model and pump manufacturer used by the patients. All patients were treated with rapid-acting insulin analogs: $52(65.8 \%)$ patients with insulin lispro, 21 (26.6\%) patients with insulin aspart, and six (7.6\%) patients with insulin glulisine. The patients were using the following models of insulin pump: MiniMed Paradigm 715 (Medtronic) $-n=16 \quad(20.2 \%)$, MiniMed Paradigm 722 (Medtronic) $-n=28$ (35.4\%), Medtronic MiniMed Veo (Medtronic) $-n=9$ (11.4\%), MiniMed 640G (Medtronic) $-n=7$ (8.9\%), Accu-Chek Spirit Combo (Roche Diabetes Care) $-n=18 \quad(22.8 \%)$, and Dana (SOOIL) $-n=1(1,2 \%)$. Most of the patients were using a Teflon cannula $(n=71,89.9 \%)$, only a small subgroup employed a steel cannula $(n=7,8.9 \%)$ (including three patients who used a Teflon and steel cannula interchangeably). The information about one patient was missing.

4.2. FH Assessment. LH was detected in 75 (94.9\%) patients diagnosed with US, in 59 (74.7\%) individuals LH was described by palpation and in $39(49.4 \%)$ patients by visual assessment, and by physical assessment defined as visual and palpation combined in $63(79.7 \%)$ patients (Supplemental
Table 1). Two patients had none LH lesions found in all methods mentioned above. Also, 19 (24.1\%) patients had skin lesions described as spot marks (redness, scars) in areas of former cannula implantation. We compared the subgroups of patients defined according to the number of $\mathrm{LH}$ lesions ( $<5 \mathrm{LH}$ lesions and 5 or $>\mathrm{LH}$ lesions on US) as shown in Supplemental Table 2. Apart from a borderline result for BMI $\left(23.7 \pm 2.9 \mathrm{~kg} / \mathrm{m}^{2}\right.$ vs. $25.3 \pm 3.9$, respectively, $\left.p=0.05\right)$, there were no differences between the subgroups.

Subclinical LH was diagnosed in 61 patients $(77.2 \%)$, who had some LH lesions detected on US but not on physical examination. In addition, 21 patients $(26.6 \%)$ had some lesions reported as LH during the visual assessment and/or palpation that were not recognized as LH on US.

The most common area of $\mathrm{LH}$ localization was the abdomen wall: LH was found in 59 patients $(74.7 \%)$ on US, in $45(57 \%)$ by palpation, and in $34(43 \%)$ by visual assessment. Two (2.5\%) patients had subcutaneous atrophy in insulin-injected areas.

Overall, by visual assessment we detected 72 singular LH lesions, $134 \mathrm{LH}$ lesions by palpation, and 372 based on US $(p<=0.001)$ (Supplemental Table 3). There were $249 \mathrm{LH}$ lesions detected only in US and not by visual or palpatory assessment. There were $29 \mathrm{LH}$ lesions described in visual and/or palpatory assessment that were not visualized on US.

We identified $13(16.5 \%)$ patients with the biggest dimension of LH below or equal to $1 \mathrm{~cm}, 30(38.0 \%)$ patients had lesions greater than $1 \mathrm{~cm}$ but less or equal to $2 \mathrm{~cm}$, in 10 (12.7\%) patients LH size was more than $2 \mathrm{~cm}$ but less or equal to $3 \mathrm{~cm}$, and in $22(27.8 \%)$ patients the lesions were greater than $3 \mathrm{~cm}$. On US examination, the majority of $\mathrm{LH}$ lesions featured hyperechogenic nodules-some with small hypoechogenic areas of edema or fluid. We found the ultrasound features of lipomas in five lesions described initially as LH in physical assessments.

US identified LH lesions more frequently than physical examination, respectively: $372 \mathrm{LH}$ lesions vs. $152 \mathrm{LH}$ lesions $(p<0.001)$. There was no inter-observer agreement between the number of LH lesions diagnosed during the physical examination and on US based on Cohen Kappa $=-0.157$ [95\% CI, -0.24- -0.08]. Similarly, there was no inter-observer agreement between the number of patients diagnosed with LH in the physical examination and on US based on Cohen's Kappa $=0.129$ [95\% CI, $-0.25-0.51]$. The sensitivity of physical examination was 35.53\% [95\% CI, 30.51-40.8] (the US as a reference method), and its specificity was $0 \%$ [95\% CI, 0.00-11.94].

We did not find any differences in the use of different insulin sets and rapid acting insulins between the groups defined on the basis of number and severity score of LH (Supplemental Tables 4 and 5).

\subsection{Risk Factors of $L H$}

4.3.1. Risk Factors of LH Number. Univariate logistic regression models showed the following risk factors of developing five and more $\mathrm{LH}$ lesions visible on US: daily insulin dose above $0.7 \mathrm{IU} / \mathrm{kg}$ (OR, 3.69; 95\% CI, 1.43-10.01), 
TABle 1: Patients characteristics.

\begin{tabular}{|c|c|c|c|c|}
\hline Variable & All patients $(n=79)^{*}$ & Patients with $\mathrm{HbA}_{1 \mathrm{c}}<7 \%(n=30)$ & Patients with $\mathrm{HbA}_{1 \mathrm{c}} \geq 7 \%(n=48)$ & $P^{*}$ \\
\hline Age, years & $28(24-30.5)$ & $29(24.3-33.8)$ & $25.5(24-30)$ & 0.21 \\
\hline Men, $n(\%)$ & $40(50.6)$ & $16(53.3)$ & $23(47.9)$ & 0.82 \\
\hline Diabetes duration, years & $15.0(9-20)$ & $12(9-19)$ & $16.5(8.8-20)$ & 0.44 \\
\hline Time in CSII, years & $8.5 \pm 4.8$ & $9.2 \pm 5.1$ & $8.2 \pm 4.6$ & 0.37 \\
\hline $\mathrm{HbA}_{1 \mathrm{c}}, \% ; \mathrm{mmol} / \mathrm{mol}$ & $7.1(6.7-8.1) 54(51-65)$ & $6.5(6.3-6.8) 47.5(45.4-50.8)$ & $7.7(7.3-8.8) 60.7(56.3-72.7)$ & $<0.001$ \\
\hline BMI, $\mathrm{kg} / \mathrm{m}^{2}$ & $24.5 \pm 3.5$ & $24.2 \pm 3.2$ & $24.7 \pm 3.7$ & 0.54 \\
\hline MDI before insulin pump, years & $3(2-8)$ & $2(1-5.8)$ & $4(2-12.3)$ & 0.01 \\
\hline Hypothyroidism, $n(\%)$ & $14(17.7)$ & $5(16.7)$ & $8(16.3)$ & 0.99 \\
\hline$\geq 5$ LH lesions on US, $n(\%)$ & $41(51.8)$ & $13(43.3)$ & $24(58.3)$ & 0.29 \\
\hline
\end{tabular}

$\mathrm{HbA}_{1 c}$, hemoglobin $\mathrm{A}_{1 c}$; CSII, continuous subcutaneous insulin infusion; BMI, body mass index; MDI, multiply insulin injection; LH, lipohyperthropy. ${ }^{*}$ For the comparison of patients with $\mathrm{HbA}_{1 \mathrm{c}}<7 \%$ and $\geq 7 \%$.

higher total daily insulin dose (OR, 1.05; 95\% CI, 1.02-1.09), and higher BMI (OR, 1.14; 95\% CI, 1.00-1.31-borderline significance). Multivariate logistic regression model revealed that a dose of insulin above $0.7 \mathrm{IU}$ per $\mathrm{kg}$ remained a significant risk factor (OR, 3.37; 95\% CI, 1.27-9.31) (Table 2).

4.3.2. Risk Factors of LH Severity. The advancement of $\mathrm{LH}$ according to the proposed scale was as follows: no LH in 4 $(5.05 \%)$ patients, mild LH in $4(5.05 \%)$ patients, moderate LH in $41(51.9 \%)$ patients, and advanced LH in $30(38 \%)$ patients.

A univariate logistic regression model showed the following risk factors of advanced LH diagnosed by US: longer length of cannula (OR, 4.01; 95\% CI, 1.52-11.39), daily insulin dose above $0.7 \mathrm{IU} / \mathrm{kg}$ of body weight (OR, 3.89, $95 \%$ CI, 1.47-10.96), higher daily insulin dose (OR, 1.03, 95\% CI, 1.004-1.06), and higher BMI (OR, 1.22; 95\% CI, 1.06-1.43). Multivariate logistic regression model revealed that the daily insulin dose above $0.7 \mathrm{IU} / \mathrm{kg}$ (OR, 4.81; 95\% CI, 1.53-17.22) and length of cannula (OR, 3.44; 95\% CI 1.11-11.74) were independent risk factors of the LH advancement (Table 3).

\section{Discussion}

This is the first study to assessing the LH of subcutaneous tissue based on physical examination and US in a selected cohort of young T1DM patients treated with a personal insulin pump. Scientific data on the LH in insulin pump users with T1DM is limited [26-29]. We confirmed previous findings from clinically diverse diabetic cohorts - LH is a common complication of insulin therapy in T1DM patients. We confirmed that for LH detection US is much more sensitive method than physical examination. Moreover, we defined several risk factors of LH number. Finally, we proposed a new scale for LH severity and assessed risk factors for advanced $\mathrm{LH}$.

Previous meta-analysis reported that the prevalence of LH in patients with T1DM was $34 \%$ based on studies assessing LH by sight and palpation. In individual studies, this prevalence differed depending on patients' characteristic and the LH detection method $[19,20]$. The LH prevalence assessed by palpation in studies involving patients on insulin pump therapy was of $44 \%$ [26]. Another study showed that $76 \%$ patients had LH assessed on US [29]. Our US-based research shows a markedly higher LH prevalence in T1DM patients treated with CSII and emphasizes the importance of LH screening in these patients.

The superiority of US over palpation was also associated with the fact that we could distinguish the LH from other subcutaneous lesions, e.g. lipoma. Some subjects had LH found with only US. These cases were undetectable in physical examination. Thus, the number of LH lesions in physical examination was substantially underestimated versus US as in previous studies [22,30].

US seems to be currently a gold standard in LH detection [31]. One of the advantages of US examination over physical examination is the fact, that it enables precise assessment of LH size. Nevertheless, a physical examination is less timeconsuming and less expensive than US and can be performed at every outpatient visit. Thus, the clinical value of these methods should not be underestimated.

We found that the dose of insulin per kilogram body weight was a LH risk factor on US - this agrees with other studies in which LH was associated with higher doses of insulin $[2,10,32]$. We also report for the first time that the insulin dose per kilogram was associated with LH severity.

The causative relationships in this observational study could not be established, but we have two potential explanations. First, insulin absorption in the area of $\mathrm{LH}$ is impaired, which forces patient to escalate insulin doses [9]. Alternatively, higher doses of insulin in the subcutaneous tissue caused LH. The length of cannula was another risk factor for LH severity. In previous studies involving patients treated with multiple daily injections, the needle length was reported to be a risk factor of $\mathrm{LH}$ in univariate analysis, but it lost significance in multivariate analysis $[2,3,33]$. There are no previous studies involving patients on insulin pump therapy in terms of association of cannula length and LH. No difference was found in insulin set or rapid acting analog use between the subgroups defined based on the number and severity score of LH. Of note, this was an observational study including one-point data and the number of patients in analyzed subgroups was rather limited. Thus, we are not able to definitely exclude the impact of specific insulin, equipment or supplies on the incidence of LH.

The BMI turned out not to be an independent risk factor of LH number and LH severity in multivariate models, and previous results are inconclusive $[3,7,15,32]$. 
TABLE 2: Univariate and multivariate models for risk factors of 5 and more LH lesions.

\begin{tabular}{|c|c|c|c|c|c|}
\hline \multirow{2}{*}{ Variable } & \multirow{2}{*}{ OR per } & \multicolumn{2}{|c|}{ Univariate } & \multicolumn{2}{|c|}{ Multivariate } \\
\hline & & OR $(95 \% \mathrm{CI})$ & $P$ & OR $(95 \% \mathrm{CI})$ & $P$ \\
\hline Age & 1 year & $1.003(0.95-1.06)$ & 0.91 & & \\
\hline Male sex & No/Yes & $0.95(0.39-2.31)$ & 0.91 & & \\
\hline BMI & $\mathrm{kg} / \mathrm{m}^{2}$ & $1.14(1.00-1.31)$ & 0.055 & $1.14(0.98-1.33)$ & 0.09 \\
\hline Diabetes duration & 1 year & $1.00(0.94-1.06)$ & 0.95 & & \\
\hline Duration of CSII & 1 year & $1.02(0.93-1.13)$ & 0.60 & & \\
\hline Regular changing of infusion sites & No/Yes & $0.73(0.21-2.44)$ & 0.61 & & \\
\hline Changing infusion set every 3 day or more frequently & No/Yes & $1.35(0.55-3.32)$ & 0.51 & & \\
\hline Length of cannula $\geq 9 \mathrm{~mm}$ & No/Yes & $1.59(0.65-3.96)$ & 0.31 & & \\
\hline $\mathrm{HbA}_{1 \mathrm{c}}$ & $\%$ & $1.23(0.85-1.84)$ & 0.29 & & \\
\hline Dose of insulin $>0.7 \mathrm{IU} / \mathrm{kg}$ & No/Yes & $3.69(1.43-10.01)$ & 0.008 & $3.37(1.27-9.31)$ & 0.016 \\
\hline Dose of insulin & IU & $1.05(1.02-1.09)$ & 0.002 & & \\
\hline
\end{tabular}

In the multivariate logistic regression model the variables with $p$-value $<0.10$ based on univariate logistic models were included (except for daily insulin dose because of including dose of insulin per kg body weight). OR, odds ratio; CI, confidence interval; for other abbreviations see Table 1.

TABLE 3: Univariate and multivariate models for risk factors of advanced LH based on the authorial scale (6-8 points).

\begin{tabular}{|c|c|c|c|c|c|}
\hline \multirow{2}{*}{ Variable } & \multirow{2}{*}{ OR per } & \multicolumn{2}{|c|}{ Univariate } & \multicolumn{2}{|c|}{ Multivariate } \\
\hline & & OR $(95 \% \mathrm{CI})$ & $P$ & OR $(95 \% \mathrm{CI})$ & $P$ \\
\hline Age & 1 year & $1.04(0.98-1.09)$ & 0.18 & & \\
\hline Male sex & No/Yes & $1.29(0.52-3.24)$ & 0.58 & & \\
\hline BMI & $\mathrm{kg} / \mathrm{m}^{2}$ & $1.22(1.06-1.43)$ & 0.007 & $1.14(0.96-1.37)$ & 0.15 \\
\hline Diabetes duration & 1 year & $1.05(0.99-1.12)$ & 0.08 & $1.06(0.99-1.14)$ & 0.13 \\
\hline Duration of CSII & 1 year & $0.99(0.90-1.09)$ & 0.82 & & \\
\hline Regular changing of infusion sites & No/Yes & $0.67(0.17-2.28)$ & 0.53 & & \\
\hline Changing infusion set every 3 day or more frequently & No/Yes & $0.84(0.33-2.11)$ & 0.72 & & \\
\hline Length of cannula $\geq 9 \mathrm{~mm}$ & No/Yes & $4.01(1.52-11.39)$ & 0.007 & $3.44(1.11-11.74)$ & 0.038 \\
\hline $\mathrm{HbA}_{1 \mathrm{c}}$ & $\%$ & $1.11(0.76-1.61)$ & 0.57 & & \\
\hline Dose of insulin $>0.7 \mathrm{IU} / \mathrm{kg}$ & No/Yes & $3.89(1.47-10.96)$ & 0.008 & $4.81(1.53-17.22)$ & 0.010 \\
\hline Dose of insulin & IU & $1.03(1.004-1.06)$ & 0.03 & & \\
\hline
\end{tabular}

In the multivariate logistic regression model the variables with $p$-value $<0.10$ based on univariate logistic models were included (except for daily insulin dose because of including dose of insulin per kg body weight). For abbreviations see Tables 1 and 2.

Some study limitations should be acknowledged. The sample size of this study is rather limited. Moreover, the causative relationships between variables could not be established due to the observational nature of this research. Additionally, T1DM duration differs substantially between the study participants; some patients are quite new to the pump. We also did not validate the clinical utility of the newly proposed severity scale. Finally, in spite of conducted LH severity assessment, a potential weakness of our study may be a lack of precise LH volume calculation in every patient.

The strength of our study is the homogeneity in the subjects in terms of the diabetes type, i.e., only CSII-treated T1DM patients were included. We also controlled for insulin analog use, severe chronic complications of diabetes, and obesity.

We suggest that assessment of LH on US should be first reserved to patients without LH lesions in clinical examination but with a high dose of insulin per kg. Moreover, patients should be educated about $\mathrm{LH}$, its risk factors, consequences, and correct habits for infusion set insertion (site rotation, regular changing infusion sets, or omission of LH areas). Less than half of diabetes patients had declared being informed about the strategies how to prevent LH development [34].
In summary, this selected T1DM cohort treated with CSII had a very high prevalence of LH. US assessment can be a reference method for $\mathrm{LH}$ screening in T1DM patients. The independent risk factors of developing 5 and more LH lesions were related to insulin dosing.

\section{Data Availability}

The data are available upon reasonable request from the corresponding author (b.matejko@uj.edu.pl).

\section{Conflicts of Interest}

The authors declare that they have no conflicts of interest.

\section{Authors' Contributions}

MT. M., T. K, D. U., S. M., and B. M. conceptualized the study; MT. M, T. K, D. U., S. M., A. G., and B. M. developed the methodology; B. M. handled software; B. M. carried out formal analysis; B. M., S. M., and D. U. carried out investigation; D. U., S. M., A. G., B. M.; collected the resources T. K.; D. U. and S. M. curated the data; S. M. and D. U. prepared the original draft; S. M., D. U., A. G., T. K., and MT. M. 
reviewed and edited the manuscript; B. M., T. K., and MT. M. supervised the study; D. U. and S. M carried out project administration.

\section{Acknowledgments}

This work was supported by Jagiellonian University Medical College, Krakow, Poland.

\section{Supplementary Materials}

Supplemental Table 1. The number of T1DM patients diagnosed with any $\mathrm{LH}$ lesion according to the diagnostic method. Supplemental Table 2. The comparison of the patients with less than $5 \mathrm{LH}$ lesions and patients with 5 or more LH lesions on US. Supplemental Table 3. The comparison of the number of any lipohypertrophic (LH) lesions according to the diagnostic method in type 1 diabetes (T1DM) patients $(n=79)$. Supplemental Table 4 . The comparison of the number of LH lesions and LH severity score according to the type of infusion set used by T1DM patients. Supplemental Table 5. The comparison of the number of LH lesions and $\mathrm{LH}$ severity score according to the insulin used by T1DM patients. (Supplementary Materials)

\section{References}

[1] T. Richardson and D. Kerr, "Skin-related complications of insulin therapy," American Journal of Clinical Dermatology, vol. 4, no. 10, pp. 661-667, 2003.

[2] A. Barola, P. Tiwari, A. Bhansali, S. Grover, and D. Dayal, "Insulin-related lipohypertrophy: lipogenic action or tissue trauma?" Frontiers in Endocrinology, vol. 9, p. 638, 2018.

[3] A. A. Al Hayek, A. A. Robert, R. B. Braham, and M. A. Al Dawish, "Frequency of lipohypertrophy and associated risk factors in young patients with type 1 diabetes: a crosssectional study," Diabetes Therapy, vol. 7, no. 2, pp. 259-267, 2016.

[4] B. Vardar and S. Kizılc1, "Incidence of lipohypertrophy in diabetic patients and a study of influencing factors," Diabetes Research and Clinical Practice, vol. 77, no. 2, pp. 231-236, 2007.

[5] M. Blanco, M. T. Hernández, K. W. Strauss, and M. Amaya, "Prevalence and risk factors of lipohypertrophy in insulininjecting patients with diabetes," Diabetes \& Metabolism, vol. 39, no. 5, pp. 445-453, 2013.

[6] C. De Coninck, A. Frid, R. Gaspar et al., "Results and analysis of the 2008-2009 insulin injection technique questionnaire survey," Journal of Diabetes, vol. 2, no. 3, pp. 168-179, 2010.

[7] F. Bertuzzi, E. Meneghini, E. Bruschi, L. Luzi, M. Nichelatti, and O. Epis, "Ultrasound characterization of insulin induced lipohypertrophy in type 1 diabetes mellitus," Journal of Endocrinological Investigation, vol. 40, no. 10, pp. 1107-1113, 2017.

[8] K. Raile, V. Noelle, R. Landgraf, and H. Schwarz, "Insulin antibodies are associated with lipoatrophy but also with lipohypertrophy in children and adolescents with type 1 diabetes," Experimental and Clinical Endocrinology \& Diabetes, vol. 109, no. 08, pp. 393-396, 2001.

[9] R. J. Young, W. J. Hannan, B. M. Frier, J. M. Steel, and L. J. P. Duncan, "Diabetic lipohypertrophy delays insulin absorption,” Diabetes Care, vol. 7, no. 5, pp. 479-480, 1984.
[10] A. H. Frid, L. J. Hirsch, A. R. Menchior, D. R. Morel, and K. W. Strauss, "Worldwide injection technique questionnaire study: injecting complications and the role of the professional," Mayo Clinic Proceedings, vol. 91, no. 9, pp. 1224-1230, 2016.

[11] T. A. Chowdhury and V. Escudier, "Poor glycaemic control caused by insulin induced lipohypertrophy," BMJ, vol. 327, no. 7411 , pp. 383-384, 2003.

[12] I. Martín-Timón, C. Sevillano-Collantes, A. Segura-Galindo, and F. J. Del Cañizo-Gómez, "Type 2 diabetes and cardiovascular disease: have all risk factors the same strength?" World Journal of Diabetes, vol. 5, no. 4, pp. 444-470, 2014.

[13] H. Arda Sürücü and H. OKurArslan, "Lipohypertrophy in individuals with type 2 diabetes: prevalence and risk factors," Journal of Caring Sciences, vol. 7, no. 2, pp. 67-74, 2018.

[14] S. Gentile, F. Strollo, G. Guarino et al., "Why are so huge differences reported in the occurrence rate of skin lipohypertrophy? Does it depend on method defects or on lack of interest?" Diabetes \& Metabolic Syndrome: Clinical Research \& Reviews, vol. 13, no. 1, pp. 682-686, 2019.

[15] L. Ji, Z. Sun, Q. Li et al., "Lipohypertrophy in China: prevalence, risk factors, insulin consumption, and clinical impact," Diabetes Technology \& Therapeutics, vol. 19, no. 1, pp. 61-67, 2017.

[16] E. T. Wei, E. Koh, M. S. Kelly, L. A. Wright, and T. S. Tylee, "Patient errors in use of injectable antidiabetic medications: a need for improved clinic-based education," Journal of the American Pharmacists Association, vol. 60, no. 5, pp. e76-e80, 2020.

[17] I. A. Harsch, "Extent of lipohypertrophy in diabetic patientsSometimes much more than meets the eye," Clinical Case Reports, vol. 7, no. 9, pp. 1813-1814, 2019.

[18] R. A. Sahasrabudhe, T. Y. Limaye, and V. S. Gokhale, "Unexplained persistent hyperglycaemia in a type I diabetes patient - is injection site lipohypertrophy the cause?" Journal of Clinical and Diagnostic Research, vol. 10, no. 9, pp. OD05-OD06, 2016.

[19] N. Deng, X. Zhang, F. Zhao, Y. Wang, and H. He, "Prevalence of lipohypertrophy in insulin-treated diabetes patients: a systematic review and meta-analysis," Journal of Diabetes Investigation, vol. 9, no. 3, pp. 536-543, 2017.

[20] H. Abu Ghazaleh, R. Hashem, A. Forbes et al., "A systematic review of ultrasound-detected lipohypertrophy in insulinexposed people with diabetes," Diabetes Therapy, vol. 9, no. 5, pp. 1741-1756, 2018.

[21] N. Taleb, V. Messier, S. Ott-Braschi, J.-L. Ardilouze, and R. Rabasa-Lhoret, "Perceptions and experiences of adult patients with type 1 diabetes using continuous subcutaneous insulin infusion therapy: results of an online survey," Diabetes Research and Clinical Practice, vol. 144, pp. 42-50, 2018.

[22] J. E. Kapeluto, B. W. Paty, S. D. Chang, and G. S. Meneilly, "Ultrasound detection of insulin-induced lipohypertrophy in Type 1 and Type 2 diabetes," Diabetic Medicine, vol. 35, no. 10, pp. 1383-1390, 2018.

[23] World Health Organization, Classification of Diabetes MellitusWorld Health Organization, Geneva, Switzerland, 2019, https://www.who.int/publications/i/item/classification-ofdiabetes-mellitus.

[24] B. Matejko, A. Kukułka, B. Kieć-Wilk, A. Stąpór, T. Klupa, and M. T. Malecki, "Basal insulin dose in adults with type 1 diabetes mellitus on insulin pumps in real-life clinical practice: a single-center experience," Advances in Medicine, vol. 2018, pp. 1-5, 2018. 
[25] R. Core Team, R: A Language and Environment for Statistical Computing.R Foundation for Statistical Computing, Vienna, Austria, 2016, https://www.R-project.org/.

[26] L. S. Conwell, E. Pope, A. M. Artiles, A. Mohanta, A. Daneman, and D. Daneman, "Dermatological complications of continuous subcutaneous insulin infusion in children and adolescents," The Journal of Pediatrics, vol. 152, no. 5, pp. 622-628, 2008.

[27] A. Deeb, L. Abdelrahman, M. Tomy et al., "Impact of insulin injection and infusion routines on lipohypertrophy and glycemic control in children and adults with diabetes," $\mathrm{Di}$ abetes Therapy, vol. 10, no. 1, pp. 259-267, 2019.

[28] I. Hernar, J. Haltbakk, and A. Broström, "Differences in depression, treatment satisfaction and injection behaviour in adults with type 1 diabetes and different degrees of lipohypertrophy," Journal of Clinical Nursing, vol. 26, no. 23-24, pp. 4583-4596, 2017.

[29] E. Patrakeeva, E. Mokhova, A. Zalevskaya, A. Tiselko, and E. Shelayeva, "Post-injection lipohypertrophy in T1DM patients using continuous insulin infusion (CSII) and multiple daily injections (MDI)," Diabetes Technology \& Therapeutics, vol. 16, pp. A154-A155, 2014.

[30] N. Volkova, I. Davidenko, J. Rudakova, and A. Sesukina, "Insulin-induced lipohypertrophy diagnostics in diabetic patients: subcutaneous fat ultrasonography: EP351," in Proceedings of the 17th European Congress of Endocrinology, vol. 37, Dublin, Ireland, May 2015.

[31] S. Gentile, G. Guarino, G. Guarino, A. Giancaterini, P. Guida, and F. Strollo, "A suitable palpation technique allows to identify skin lipohypertrophic lesions in insulin-treated people with diabetes," SpringerPlus, vol. 5, no. 1, p. 563, 2016.

[32] M. A. Omar, A. A. El-Kafoury, and R. I. El-Araby, "Lipohypertrophy in children and adolescents with type 1 diabetes and the associated factors," BMC Research Notes, vol. 4, no. 1, p. 290, 2011.

[33] A. H. Frid, G. Kreugel, G. Grassi et al., "New insulin delivery recommendations," Mayo Clinic Proceedings, vol. 91, no. 9, pp. 1231-1255, 2016.

[34] K. Strauss, H. D. Gols, I. Hannet, T.-M. Partanen, and A. Frid, "A pan-European epidemiologic study of insulin injection technique in patients with diabetes," Practical Diabetes International, vol. 19, no. 3, pp. 71-76, 2002. 